\title{
Power Transformers Diagnostics using Heat Model Parameter Identification
}

\author{
Prytyskach Ivan ${ }^{1}$, Yaremenko Artem ${ }^{2}$ \\ Power Supply Department, National Technical University of Ukraine "Igor Sikorsky Kyiv Polytechnic Institute", \\ Kiev, Ukraine ${ }^{1}$
}

\begin{abstract}
Condition evaluation of the electrical equipment in operation is an important prerequisite for building reliable and efficient electrical systems and networks. In order to increase accuracy of power transformers condition monitoring, method for analyzing the diagnostic parameters of power transformer oil temperature have been developed. The method is based on parametric identification of the thermal model using particle swarm optimization. In order to test the efficiency of the proposed method, simulation was performed using the experimental data obtained on the power three-winding transformer of the network substation. In view of the impossibility of deliberately changing the technical condition of the transformer, the performance evaluation is based on the confirmation of the absence of changes in the technical state. The analysis of the obtained results confirms the efficiency of the developed method and algorithms.
\end{abstract}

Keywords: power transformer, diagnostic parameter, oil temperature in the upper layers, condition estimation, parameter identification.

\section{INTRODUCTION}

In today's world, it becomes obvious that to improve the efficiency of the electric power companies, it is urgent to shift to a more progressive strategy of operational reliability maintenance. This strategy is based on the repair measures that are carried out on the actual condition of electrical equipment [1]. This requires reliable and timely information on the technical condition of electrical equipment such as power transformers. To perform this objective, power transformer diagnostic systems is used [2]. They can determine the current status of all transformer equipment using all available diagnostic parameters.

Current level of measuring instruments development makes it possible to obtain information about the meaning of most of the parameters of electrical equipment directly in the process of its operation under load [3]. Modern hardware allows to automatically measure the primary diagnostic parameters under operating voltage by using sensors installed directly on the transformer body. Application of permanent condition monitoring enables even to detect defects that are developing rapidly, with a period of several minutes. Therefore, the problem of improving the technical condition identification methods of the power transformers is very important in the current electrical networks. Research associated with the development of such methods in carried out in large number of studies [4-7].

\section{PROPOSED METHODOLOGY}

To assess the technical condition of power transformers in operating modes, it is convenient to compare the current values of the diagnostic parameters with the base data [8]. In order to identify reliable indicators of deterioration of the transformer condition, it is suggested to determine the statistical characteristics of a random process $v(t)$ for a certain base time $T_{\mathrm{b}}$, which ensure smoothing of sudden fluctuations and underscore long-term trends. The measurement of the primary diagnostic parameters occurs at a sampling rate in time, so the formulas for determining the derivatives of the diagnostic parameters are conveniently written in a discrete form.

The mathematical model for determining the basic values of the diagnostic parameter is:

$$
x_{i . M}=f\left([\mathbf{u}]_{i},[\lambda]\right)
$$

where $x_{i, M}$ is the value of the diagnostic parameter, which was simulated at the $\mathrm{i}$-th sampling interval; $[\mathbf{u}]_{i}$ are input variables of the model; $[\lambda]$ are coefficients of the model. 
Deviation of the measured value is calculated as:

$$
v_{i}=\frac{x_{i . P}-x_{i . M}}{x_{i . M}},
$$

where $x_{i . P}$ is the value of the diagnostic parameter measured at the $\mathrm{i}$-th sampling interval.

Proposed derived diagnostic parameter are:

- $\quad$ quantile with probability $\alpha_{i}$ :

$$
v_{i, \alpha}=\inf \left\{\psi \in \mathbf{R}: C D F_{i}(\psi) \geq \alpha_{v}\right\},
$$

where $C D F(\psi, t)$ - is the distribution function

$$
C D F_{i}(\psi)=p\left(\left|v_{i . k}\right| \leq \psi, k=\left(i-N_{b . \alpha}\right) \ldots i\right) .
$$

- $\quad$ angular coefficient of the linear trend

$$
v_{i . \delta}=\frac{12}{N_{b . \delta}}\left(\frac{1}{2} \sum_{k=i-N_{b, \delta}}^{i} v_{k}-\frac{1}{N_{b . \delta}} \sum_{k=i-N_{b, \delta}}^{i}(i-k) v_{k}\right) .
$$

Model for determining the basic values of diagnostic parameters generally contain a certain set of coefficients $\Lambda$. The main task of parametric identification of this model is to find the set of model coefficients $[\lambda]_{\text {opt }}$ from the solution space $\Omega$, for a typical load mode of the transformer, which minimizes the total error between the measured and modeled values of the diagnostic parameters [9]. This task can be considered as minimization without restrictions for the objective function, defined as a weighted sum of estimation errors:

$$
\left\{\begin{array}{c}
\min _{\Lambda \in \Omega} f([\lambda]) ; \\
f([\lambda])=\sum_{i} w_{i}\left(x_{i . P}-x_{i, M}\left([\mathbf{u}]_{i},[\lambda]\right),\right)^{2} .
\end{array}\right.
$$

To solve the optimization problem, the particle swarm method [10] was used, which optimizes the function, supporting the population of possible solutions (the so-called "particles") and moving these particles in the solution space. Each particle in the swarm corresponds to its coordinates $[\lambda]_{i}$, velocity $[\mathbf{v}]_{i}$, and the best-known position of this particle $[\mathbf{p}]_{i}$ from the solution space $\Omega$. Particles moving obey the principle of the best position found in this space, the situation constantly changes when the particles find advantageous positions.

The differential equation for the process of temperature change in the upper oil layers has the following form [9]:

$$
\Delta \theta_{o}=k_{11} \tau_{0} \frac{d \theta_{o}}{d t}+\theta_{o}-\theta_{a},
$$

where $\theta_{o}$ is the oil temperature; $\theta_{a}$ is the ambient temperature; $\Delta \theta_{o}$ is the excess of the oil temperature above the ambient temperature; $\tau_{0}$ is the time constant for heating the oil; $k_{11}$ is the - coefficient, depending on the power and type of cooling of the transformer.

In [9] it is suggested to use the difference form of the notation of equation (7), which has the following form:

$$
D \theta_{o}=\frac{D t}{k_{11} \tau_{0}}\left[\Delta \theta_{o r}\left(\frac{1+R K^{2}}{1+R}\right)^{\eta}-\left(\theta_{o}-\theta_{a}\right)\right],
$$

where $K$ is the load factor of the transformer; $\Delta \theta_{\text {or }}$ is the excess oil temperature at maximum load; $\mathrm{R}$ is the ratio of short-circuit losses to idle losses; $\eta$ is the exponent of the oil.

At each time interval, the value $D \theta_{o}$ is calculated on the basis of (n-1)-th value according to the formula:

$$
\theta_{o(n)}=\theta_{o(n)}+D \theta_{o(n)} \text {. }
$$


It is proposed to fill the set of model coefficients that are used to parametric identification by such values as excess of oil temperature at maximum load $\Delta \theta_{\text {or }}$; the ratio of short-circuit losses to idle losses R; oil time constant $\tau_{0}$ and exponent of the oil $\eta$ :

$$
\lambda_{\theta}=\left\{\begin{array}{llll}
\tau_{o} & \Delta \theta_{\text {or }} & R & \eta
\end{array}\right\} .
$$

\section{SIMULATION RESULTS}

The effectiveness of the proposed method is estimated using the data measured on the operating transformer with cooling type OF. The calculations were performed using the mathematical package MATLAB. The results of the simulation and parametric identification are presented in Table. 1. On the basis of parametric identification the coefficients included in the set are refined using the data measured in the normal operating mode of the transformer. Refined values of thermal characteristics allowed to reduce by $5 \%$ the average deviation of the measured values from the simulated ones.

TABLE I RESULTS OF THE TRANSFORMER THERMAL CHARACTERISTICS A MODELLING

\begin{tabular}{|l|l|l|l|l|}
\hline & $\begin{array}{l}\text { Nominal excess of oil temperature } \\
\text { in the upper layers } \square \square_{\text {or }},{ }^{\circ} \mathbf{C}\end{array}$ & $\begin{array}{l}\text { Ratio of } \\
\text { losses R }\end{array}$ & $\begin{array}{l}\text { Oil } \\
\text { exponent, } \boldsymbol{\eta}\end{array}$ & $\begin{array}{l}\text { Time constant } \\
\boldsymbol{\tau}_{0}, \text { min }\end{array}$ \\
\hline Nominal value & 56 & 6 & 1 & 90 \\
\hline Calculated value & 48.6 & 9.7 & 0.9 & 112 \\
\hline Relative difference, \% & 13.2 & -61.7 & 10.0 & -24.4 \\
\hline
\end{tabular}

After the parametric identification of the thermal model, a calculation of the diagnostic parameters for evaluating the transformer condition has been performed. As experimental data, the recordings of the normal operating modes of the transformer were used, which were recorded by data acquisition system.

The load factor was calculated as

$$
K_{i}=\frac{S_{i}}{S_{\mathrm{n}}},
$$

where $S_{\mathrm{n}}=31500 \mathrm{kVA}$ is the rated power of the transformer. The obtained values of the load factor are shown in Fig. 1.

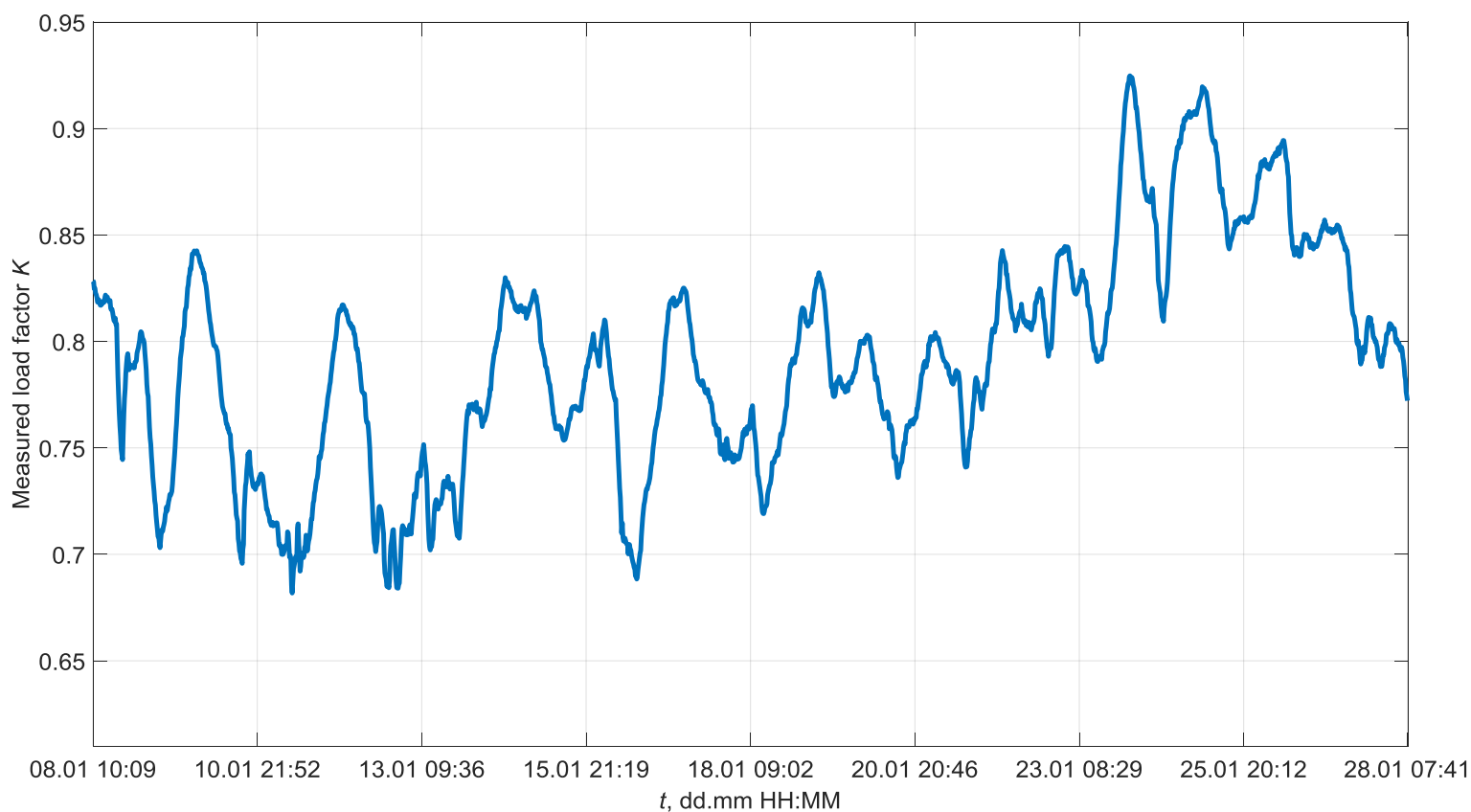

Fig. 1 Load factor 
The value of the oil temperature does not exceed $40{ }^{\circ} \mathrm{C}$, which can be explained by the insignificant load of the transformer, and also by the low temperature of the cooling air in the period under consideration.

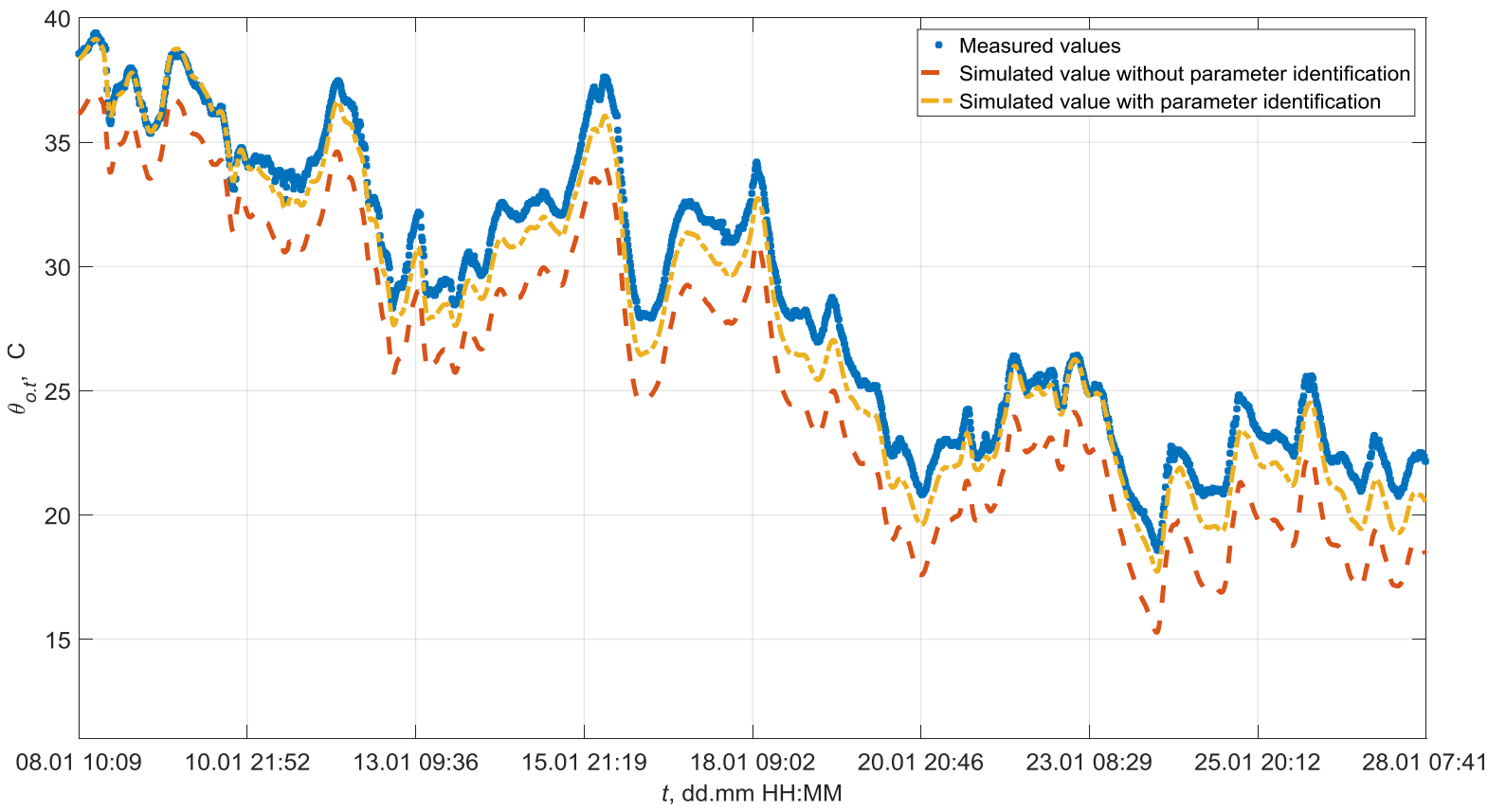

Fig. 2 Oil temperature simulation results

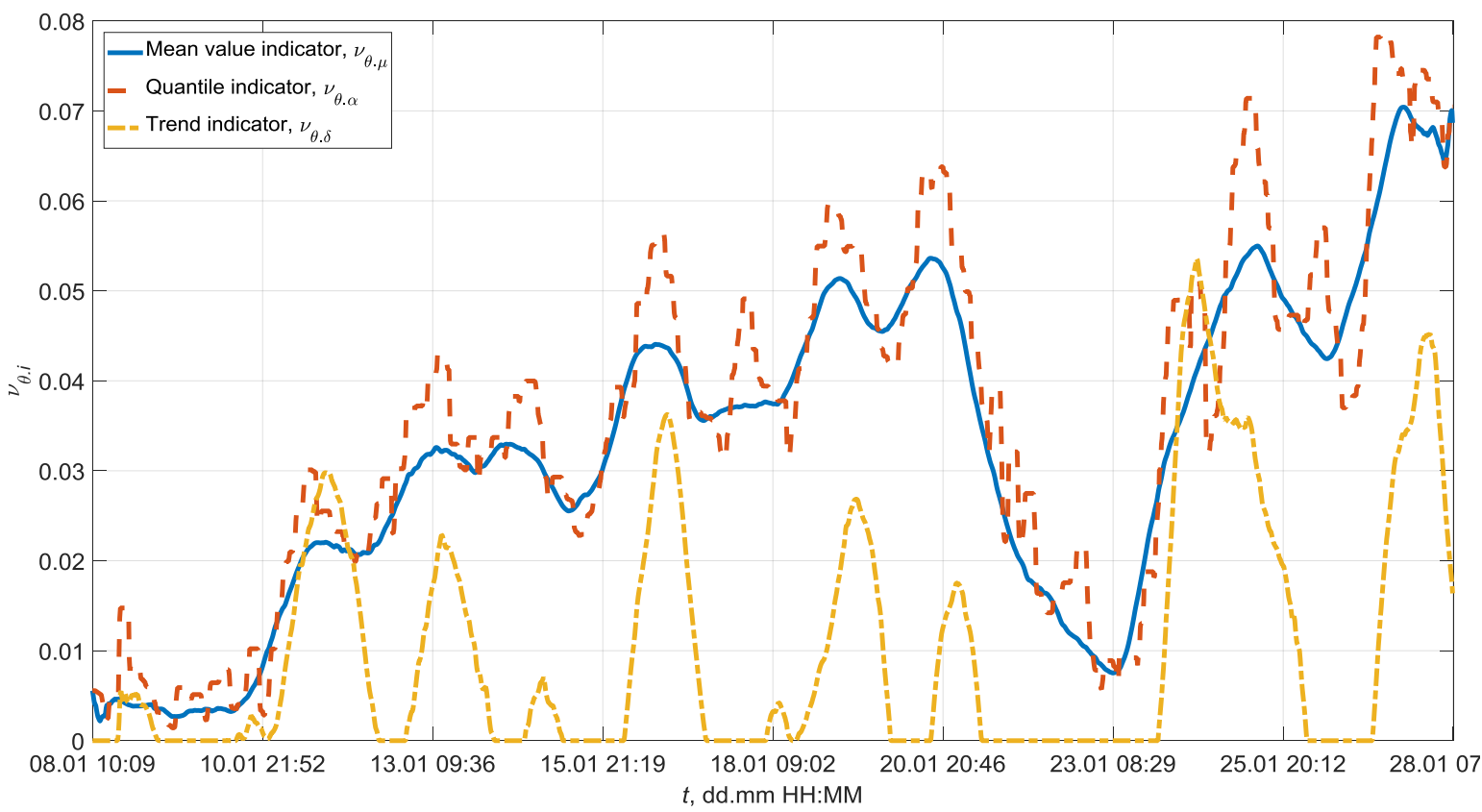

Fig. 3 Dependence of diagnostic parameters for oil temperature on time

Fig. 3 shows the diagnostic parameters of the oil temperature, obtained by (3), (5). As we can see, the absolute values do not exceed 0.08 , which indicates that there are no signs of deterioration in terms of the thermal processes in the transformer over the considered period of time.

The analysis of the obtained results of the calculation of the diagnostic parameters for the oil temperature confirms the effectiveness of procedures for reducing the effect of random errors and the efficiency of the developed algorithms and indicates the possibility of their use with data obtained with sensors mounted on the transformer. 


\section{IJIREEICE

\section{CONCLUSION}

A mathematical model for diagnostic parameters analysis of the oil temperature of a power transformer is developed, which is based on parametric identification of the thermal model by the particle swarm method. The use of the developed model in the of power transformers diagnostic system will improve the accuracy of detecting the defects of the transformer in operating conditions.

\section{REFERENCES}

[1] M. Wang, A.J Vandermaar, K.D Srivastava, 2002, "Review of Condition Assessment of Power Transformers in Service", IEEE Electrical Insulation Magazine, Vol. 18, No. 6.

[2] Saha, Tapan K. "Review of modern diagnostic techniques for assessing insulation condition in aged transformers." IEEE transactions on dielectrics and electrical insulation, 2003, vol. 10.5, pp. 903-917.

[3] Catterson, V. M., et al. "On-line transformer condition monitoring through diagnostics and anomaly detection." Intelligent System Applications to Power Systems, 2009. ISAP'09. 15th International Conference on. IEEE, 2009.

[4] A. G. Deakin, J. W. Spencer et al., "Chromatic Optoacoustic Monitoring of Transformers and their on load Tap Changers", IEEE Trans. Power Del., 2014, vol. 29, no. 1, pp. $207-214$.

[5] Y. M. Zheng, Z. Wang, "Study on broadband loss characteristics of oil-immersed papers for fast transient modeling of power transformer". IEEE Trans. Dielectr. Electr. Insul., 2013, vol. 20, no. 2, pp. 564 - 570.

[6] L. E. Lundgaard, W. Hansen, D. Linhjell and T. J. Painter, "Aging of oil-impregnated paper in power transformers," IEEE Trans. Power Deliv., vol. 19, pp. 230-239, Jan. 2004.

[7] N. Wang, F. C. Lu, and H. M. Li, “Analytical Processing of On-line Monitored Dissipation Factor Based on Morphological Filter," IEEE Trans. Dielec. Electr. Insul., vol. 11, pp. 840-846, March 2004.

[8] Denysiuk S., Prytyskach I. "Development of the On-line Power Transformer State Monitoring System.", International Journal of Computing and Technology", 2014, vol. 1.5, pp. 191-195.

[9] Power transformers - Part 7: Loading guide for oil-immersed power transformers, IEC 60076-7, 2005.

[10] Eberhart R.C. "Particle swarm optimization: developments, applications and resources", Proc. of the 2001 Congress on Evolutionary Computation, 2001, vol. 1. 